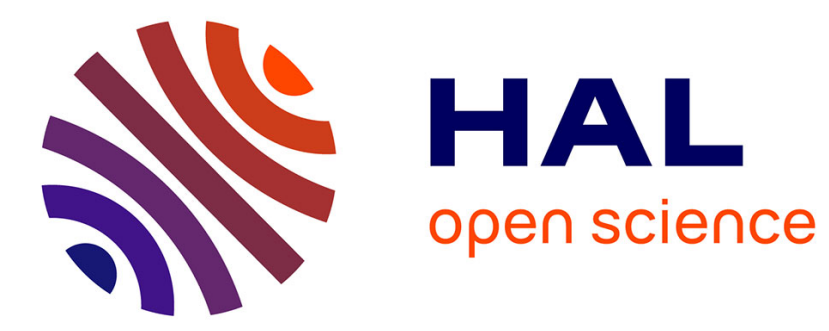

\title{
PERFORMANCE ANALYSIS OF SOME EIGEN-BASED HYPOTHESIS TESTS FOR COLLABORATIVE SENSING
}

Pascal Bianchi, Jamal Najim, Mylène Maïda, Merouane Debbah

\section{- To cite this version:}

Pascal Bianchi, Jamal Najim, Mylène Maïda, Merouane Debbah. PERFORMANCE ANALYSIS OF SOME EIGEN-BASED HYPOTHESIS TESTS FOR COLLABORATIVE SENSING. IEEE Workshop in Statistical Signal Processing (SSP-09), Aug 2009, United Kingdom. 4 p. hal-00447037

\section{HAL Id: hal-00447037 \\ https://hal-centralesupelec.archives-ouvertes.fr/hal-00447037}

Submitted on 14 Jan 2010

HAL is a multi-disciplinary open access archive for the deposit and dissemination of scientific research documents, whether they are published or not. The documents may come from teaching and research institutions in France or abroad, or from public or private research centers.
L'archive ouverte pluridisciplinaire HAL, est destinée au dépôt et à la diffusion de documents scientifiques de niveau recherche, publiés ou non, émanant des établissements d'enseignement et de recherche français ou étrangers, des laboratoires publics ou privés. 


\title{
PERFORMANCE ANALYSIS OF SOME EIGEN-BASED HYPOTHESIS TESTS FOR COLLABORATIVE SENSING
}

\author{
Pascal Bianchi ${ }^{\ddagger}$, Jamal Najim ${ }^{\ddagger}$, Mylène Maida ${ }^{\dagger}$ and Mérouane Debbah ${ }^{\dagger \dagger}$ \\ ${ }^{\ddagger}$ CNRS/LTCI Telecom Paristech, Paris, France \\ ${ }^{\dagger \dagger}$ SUPELEC, Chaire Alcatel-Lucent, Gif-sur-Yvette, France \\ †Laboratoires de Mathématiques, Université de Paris-Sud, Orsay, France \\ \{bianchi,najim\}@telecom-paristech.fr, merouane.debbah@supelec.fr, mylene.maida@normalesup.org
}

\begin{abstract}
In this contribution, we provide a theoretical study of two hypothesis tests allowing to detect the presence of an unknown transmitter using several sensors. Both tests are based on the analysis of the eigenvalues of the sampled covariance matrix of the received signal. The Generalized Likelihood Ratio Test (GLRT) derived in [1] is analyzed under the assumption that both the number $K$ of sensors and the length $N$ of the observation window tend to infinity at the same rate: $K / N \rightarrow c \in(0,1)$. The GLRT is compared with a test based on the condition number used which is used in cognitive radio applications. Using results of random matrix theory for spiked models and tools of Large Deviations, we provide the error exponent curve associated with both test and prove that the GLRT outperforms the test based on the condition number.
\end{abstract}

\section{INTRODUCTION}

In the context of cognitive networks [2], sensing is one the major of steps in order for the flexible network to adapt its parameters to the environment context. In general, the sensing procedure requires the knowledge of the noise variance as well as a high number of samples for a successful test. This is rarely compatible with the mobile constraints of the users and has pushed the community to propose alternative methods based on collaborative sensing to reduce the number of samples required [3], [4]. The techniques proposed trade the time dimension (samples) with the space dimension (antennas or base stations) and do not require the knowledge of the noise variance, which is one of the drawbacks of energy detector techniques [5]. The general idea of these new techniques compute some functionals of the eigenvalues of the sample covariance matrix which cancel out the noise variance.

The paper is articulated as follows: next Section focuses on the problem formulation and contains the signal model, while in Section III the two tests are detailed. The asymptotic analytical study is detailed in Section IV. Simulation results illustrate our claims.

\section{SIGNAL MODEL}

Consider a secondary wireless network formed by $K$ nodes, working in sensing mode. We assume that all $K$ nodes are simultaneously sensing a given sub-band $\mathcal{B}$ of the spectrum. For each $k=1, \ldots, K$, we denote by $y_{k}(n)$ the complex envelope of the signal received by the $k$ th sensor in band $\mathcal{B}$ after proper filtering and sampling. Denote by $\boldsymbol{y}(n)=\left[y_{1}(n), \ldots, y_{K}(n)\right]^{T}$ the vector obtained when stacking all $K$ sensors' observations at time $n$ into a column vector. The aim is to detect the presence of a primary transmitter in band $\mathcal{B}$. We respectively denote by $H_{0}$ and $H_{1}$ the hypotheses corresponding to the case where "band $\mathcal{B}$ is free" and "a primary device is already transmitting in band $\mathcal{B} "$ :

$$
\boldsymbol{y}(n)=\left\{\begin{array}{ll}
\boldsymbol{w}(n): & H_{0} \\
\boldsymbol{h} s(n)+\boldsymbol{w}(n): & H_{1}
\end{array},\right.
$$

where $\boldsymbol{w}(n)$ represents a complex circular temporally-white Gaussian noise vector with zero mean and covariance matrix equal to $\sigma^{2} \mathbf{I}_{K}$. In the $H_{1}$-case, vector $\boldsymbol{h} \in \mathbb{C}^{K \times 1}$ represents the complexvalued Single-Input Multiple-Output (SIMO) channel between the primary transmitter and the $K$ receiving nodes. Sequence $s(n)$ denotes the unknown data process sent by the active primary device. Sequence $s(n)$ is assumed to be an independent identically distributed (i.i.d.) zero mean random sequence. We assume without restriction that $s(n)$ has unit variance. In order to be able to derive hypothesis testing procedures and to analyze their performance in terms of probability of false alarm and power, we make the usual assumption that the transmitted symbols are Gaussian distributed, say $s(n) \sim \mathcal{C} \mathcal{N}(0,1)$. We assume that

- the noise variance $\sigma^{2}$ is unknown,

- the channel matrix $\boldsymbol{h}$ is unknown.

In the sequel, we denote by $N$ the number of samples observed by each sensor $k$. Consider the following $K \times N$ data matrix $\mathbf{Y}$ :

$$
\mathbf{Y}=[\boldsymbol{y}(0), \ldots, \boldsymbol{y}(N-1)] \text {. }
$$

In order to test hypothesis $H_{0}$ versus $H_{1}$, the aim is to construct a relevant test function $\varphi: \mathbb{C}^{K \times N} \rightarrow\{0,1\}$ with the sense that one decides hypothesis $H_{0}$ (resp. $H_{1}$ ) whenever $\varphi(\mathbf{Y})=0$ (resp. $\varphi(\mathbf{Y})=1$ ). As usual, we restrict ourselves to the search for test functions such that the probability of false alarm does not exceed a predefined level $\alpha$ i.e.,

$$
\mathbb{P}_{H_{0}}[\varphi(\mathbf{Y})=1] \leq \alpha,
$$

where $\mathbb{P}_{H_{i}}[\mathcal{E}]$ represents the probability of a given event $\mathcal{E}$ under hypothesis $H_{i}, i=0,1$. On the otherhand, the power of the test is given by $\mathbb{P}_{H_{1}}[\varphi(\mathbf{Y})=1]$.

\section{EIGEN-BASED HYPOTHESIS TESTS}

\section{III-A. Generalized Likelihood Ratio Test}

We respectively denote by $p_{0}\left(\mathbf{Y} ; \sigma^{2}\right)$ and $p_{1}\left(\mathbf{Y} ; \boldsymbol{h}, \sigma^{2}\right)$ the likelihood functions of the observation matrix $\boldsymbol{y}$ indexed by the unknown parameters $\boldsymbol{h}$ and $\sigma^{2}$ under hypotheses $H_{0}$ and $H_{1}$ respectively:

$$
\begin{aligned}
p_{0}\left(\mathbf{Y} ; \sigma^{2}\right) & =\left(\pi \sigma^{2}\right)^{-N K} \exp \left(-\frac{N}{\sigma^{2}} \operatorname{tr} \hat{\mathbf{R}}\right) \\
p_{1}\left(\mathbf{Y} ; \boldsymbol{h}, \sigma^{2}\right) & =\left(\pi^{K} \operatorname{det} \mathbf{R}\right)^{-N} \exp \left(-N \operatorname{tr}\left(\hat{\mathbf{R}} \mathbf{R}^{-1}\right)\right)
\end{aligned}
$$


where $\mathbf{R}=\mathbf{R}\left(\boldsymbol{h}, \sigma^{2}\right)$ is the true covariance matrix under $H_{1}$ defined by

$$
\mathbf{R}=\boldsymbol{h} \boldsymbol{h}^{H}+\sigma^{2} \mathbf{I}_{K}
$$

and where $\hat{\mathbf{R}}$ is the sampled covariance matrix:

$$
\hat{\mathbf{R}}=\frac{1}{N} \mathbf{Y} \mathbf{Y}^{H}
$$

In the ideal case where parameters $\boldsymbol{h}$ and $\sigma^{2}$ are supposed to be available, it is well known that a uniformly most powerful test is obtained through the Neyman-Pearson procedure, which consists in rejecting the null hypothesis for large values of the likelihood ratio statistic $p_{1}\left(\mathbf{Y} ; \sigma^{2}\right) / p_{0}\left(\mathbf{Y} ; \boldsymbol{h}, \sigma^{2}\right)$. Unfortunately, parameters $\boldsymbol{h}$ and $\sigma^{2}$ are unknown in our context so that a uniformly powerful test can no longer be that easily defined. In this case, a suboptimal but classical approach consists in replacing the true likelihood ratio by the following generalized likelihood ratio (GLR)

$$
L_{N}=\frac{\sup _{\boldsymbol{h}, \sigma^{2}} p_{1}\left(\mathbf{Y} ; \boldsymbol{h}, \sigma^{2}\right)}{\sup _{\sigma^{2}} p_{0}\left(\mathbf{Y} ; \sigma^{2}\right)} .
$$

In the GLRT procedure, one rejects hypothesis $H_{0}$ whenever $L_{N}>$ $\xi_{N}$, where $\xi_{N}$ is a certain threshold which is selected so that the probability of false alarm (3) does not exceed a given level $\alpha$. Denote by $\lambda_{1}>\lambda_{2} \cdots>\lambda_{K} \geq 0$ the ordered eigenvalues of $\hat{\mathbf{R}}$ (all distincts with probability one). As we shall see below, the GLR can be written as a function of the ratio

$$
T_{N}^{(1)}=\frac{\lambda_{1}}{\frac{1}{K} \operatorname{tr} \hat{\mathbf{R}}} .
$$

The following proposition follows from the results of [1] after straightforward manipulations.

Proposition 1. The GLR (6) writes

$$
L_{N}=\left(C T_{N}^{(1)}\left(1-\frac{T_{N}^{(1)}}{K}\right)^{K-1}\right)^{-N}
$$

where $C=\left(1-\frac{1}{K}\right)^{K-1}$ is a constant.

By Proposition 1, the GLR can be written as a function of the ratio $T_{N}^{(1)}$ between the largest eigenvalue $\lambda_{1}$ of the sampled covariance matrix $\hat{\mathbf{R}}$ and the normalized trace of $\hat{\mathbf{R}}$, say $L_{N}=$ $\phi_{N, K}\left(T_{N}^{(1)}\right)$ where $\phi_{N, K}: x \mapsto C x^{-N}\left(1-\frac{x}{K}\right)^{N(1-K)}$. Note that $T_{N}^{(1)}$ belongs to the interval $(1, K)$ with probability one and that function $\phi_{N, K}$ is increasing on this interval. The GLRT rejects the null hypothesis when inequality $L_{N}>\xi_{N}$ holds. As $\phi_{N, K}$ is increasing, the latter inequality is equivalent to $T_{N}^{(1)}>\phi_{N, K}^{-1}\left(\xi_{N}\right)$. Otherwise stated, the GLRT reduces to the test which rejects the null hypothesis for large values of $T_{N}^{(1)}$ :

$$
T_{N}^{(1)} \underset{H_{0}}{\stackrel{H_{1}}{\gtrless}} \gamma_{N}
$$

where $\gamma_{N}=\phi_{K}^{-1}\left(\xi_{N}\right)$ is a certain threshold which is such that the probability of false alarm does not exceed a given level $\alpha$. Before studying the performance of the above test, we must now complete the definition of this test by providing a practical way to set the threshold $\gamma_{N}^{(1)}$ in (8).

\section{III-B. Setting the Threshold $\gamma_{N}^{(1)}$}

In order to maximize the power of our test while keeping the PFA constraint (3) satisfied, we must select the threshold $\gamma_{N}^{(1)}$ such that $\mathbb{P}_{H_{0}}\left[T_{N}^{(1)} \leq \gamma_{N}^{(1)}\right]=\alpha$. This requires the tedious computation of the distribution function of $T_{N}^{(1)}$ under $H_{0}$ for each $N, K$. Such a computation is usually impractical in cognitive radio applications, due to complexity/delay constraints, along with the fact that the number of sensors nodes $K$ and the number of observations $N$ are frequently varying. In order to simplify the selection of $\gamma_{N}^{(1)}$, we recently investigated in [6] the asymptotic case where both the number $K$ of sensors and the number of observations are assumed to be large. In this case, simple expressions of the threshold $\gamma_{N}^{(1)}$ can be derived. More precisely, we studied the behaviour of $T_{N}^{(1)}$ under $H_{0}$ in the asymptotic regime

$$
N \rightarrow \infty, K \rightarrow \infty, K / N \rightarrow c,
$$

where $0<c<1$ is a constant. This asymptotic regime is relevant under cognitive radio constraints, as the secondary system must be able to decide the presence/absence of primary transmitters in a moderate amount of time: the number $K$ of sensors and the number $N$ of samples have therefore the same order of magnitude. In the asymptotic regime (9), it was proved in [6] that, putting $c_{N}=K / N$,

$$
N^{2 / 3}\left(\frac{T_{N}^{(1)}-\left(1+\sqrt{c_{N}}\right)^{2}}{\left(1+\sqrt{c_{N}}\right)\left(\frac{1}{\sqrt{c_{N}}}+1\right)^{1 / 3}}\right) \underset{H_{0}}{\stackrel{\mathcal{D}}{\longrightarrow}} X
$$

where $\underset{H_{0}}{\stackrel{\mathcal{D}}{\longrightarrow}}$ stands for the convergence in distribution under $H_{0}$ and where $X$ is a random variable which follows the Tracy-Widom distribution function $F_{T W}($.$) associated with the Gaussian unitary$ Ensemble (see [6] for details). As a consequence, we obtain the following result.

Proposition 2. The power of test (8) is maximum under constraint (3) only if the threshold $\gamma_{N}^{(1)}$ writes

$$
\gamma_{N}^{(1)}=\left(1+\sqrt{c_{N}}\right)^{2}+\frac{\beta_{N}^{(1)}}{N^{2 / 3}}
$$

for some $\beta_{N}^{(1)}$ which tends to $(1+\sqrt{c})\left(\frac{1}{\sqrt{c}}+1\right)^{1 / 3} F_{T W}^{-1}(1-\alpha)$.

The above Proposition was used in [6] to derive practical guidelines to select the threshold $\gamma_{N}^{(1)}$ without resorting to a tedious computation of the exact distribution function $x \mapsto \mathbb{P}_{H_{0}}\left[T_{N}^{(1)} \leq x\right]$. This result will also be useful in Section IV in order to analyze the performance of the proposed hypothesis test. Before providing such a performance analysis, we mention the existence of an other hypothesis testing approach which has been recently developed in [4], [3], [7] for cognitive radio contexts.

\section{III-C. An Other Existing Hypothesis Testing Approach}

A different approach introduced in several papers devoted to cognitive radio contexts [4], [3], [7] consists in rejecting the $H_{0^{-}}$ hypothesis when the following statistic

$$
T_{N}^{(2)}=\frac{\lambda_{1}}{\lambda_{K}}
$$

lies above a well chosen threshold $\gamma_{N}^{(2)}$. The introduction of the above statistic $T_{N}^{(2)}$ can be motivated by the following observation. Assume that the following limiting signal-to-noise ratio (SNR) is well defined :

$$
\rho=\lim _{K \rightarrow \infty} \frac{\|\boldsymbol{h}\|^{2}}{\sigma^{2}}
$$




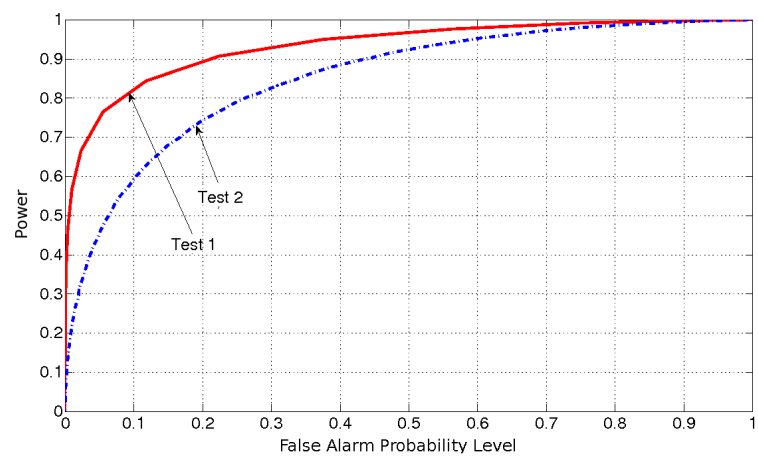

Fig. 1. ROC curves of tests $T_{N}^{(1)}$ and $T_{N}^{(2)}-K=10, N=50, \rho=1$.

where $\|\boldsymbol{h}\|$ denotes the L2-norm of the $K \times 1$ vector $\boldsymbol{h}$. Then, in the asymptotic regime (9),

$$
\begin{aligned}
& T_{N}^{(2)} \underset{H_{0}}{\stackrel{a . s .}{\longrightarrow}} \frac{(1-\sqrt{c})^{2}}{(1+\sqrt{c})^{2}} \\
& T_{N}^{(2)} \underset{H_{1}}{\stackrel{a . s .}{\longrightarrow}} \begin{cases}\frac{(1+\rho)(1+c / \rho)}{(1+\sqrt{c})^{2}} & \text { if } \rho>\sqrt{c} \\
\frac{(1-\sqrt{c})^{2}}{(1+\sqrt{c})^{2}} & \text { if } \rho<\sqrt{c} .\end{cases}
\end{aligned}
$$

Provided that the SNR is large enough $(\rho>\sqrt{c}), T_{N}^{(2)}$ converges to different values depending on the true hypothesis. This motivates the fact that the value of $T_{N}^{(2)}$ can be used to decide which hypothesis is true.

\section{III-D. Simulation Analysis}

In the following, we compare by simulations both tests in the case where $K=10, N=50$ and $\rho=1$. For a fixed level $\alpha$, the thresholds $\gamma_{N}^{1,2}$ corresponding to the probability of error under $H_{0}$ are given by $P_{H_{0}}\left(T_{N}^{(1,2)} \geq \gamma_{N}^{(1,2)}\right)=\alpha$. The power of the test is then given by $P_{H_{1}}\left(T_{N}^{(1,2)} \geq \gamma_{N}^{(1,2)}\right)$. Figure (1) provides the ROC curve for both tests. tests. It clearly shows that the test $T_{1}$ outperforms the test $T_{2}$. In the rest of the paper, we provide a theoretical performance study of the tests based on $T_{N}^{(1)}$ and $T_{N}^{(2)}$ respectively to sustain the experimental claims of Figure 1. Using large deviations arguments, we rigorously prove that the test (8) based on $T_{N}^{(1)}$ outperforms the test based on $T_{N}^{(2)}$.

\section{ERROR EXPONENTS}

\section{IV-A. Definition}

The most natural approach to characterize the performance of the tests associated with statistics $T_{N}^{(1)}$ and $T_{N}^{(2)}$ is to evalute the power of each of these tests, or equivalently the miss probability $\mathbb{P}_{H_{1}}\left(T_{N}^{(i)}<\gamma_{N}^{(i)}\right), i=1,2$. As the miss probability has no simple expression in the general case, we propose to study the asymptotic behaviour of the miss probability in the asymptotic regime (9) of interest. More precisely, for each test $i=1,2$, we prove the existence and provide the expression of the following error exponents

$$
\varepsilon_{i, \rho}=\lim _{N \rightarrow \infty}-\frac{1}{N} \log \inf \left\{\mathbb{P}_{H_{1}}\left(T_{N}^{(i)}<\gamma\right)\right\},
$$

where the infemum is taken w.r.t. all $\gamma$ such that PFA constraint (3) holds for a fixed level $\alpha$. Of course, as $N, K$ tend to infinity, one may as well take benefit of the increasing number of data in order not only to decrease the miss probability, but to decrease the PFA as well. As a consequence, it is of practical interest to analyze the detection performance when both the miss probability and the PFA tend to zero at exponential speed. A couple $(a, b) \in(0, \infty) \times$ $(0, \infty)$ is said to be an achievable pair of error exponents for the test $T_{N}^{(i)}$ if there exists a sequence of thresholds $\gamma_{N}$ such that, in the asymptotic regime (9),

$$
\begin{aligned}
& \lim _{N \rightarrow \infty}-\frac{1}{N} \log \mathbb{P}_{H_{0}}\left(T_{N}^{(i)}>\gamma_{N}\right)=a \\
& \lim _{N \rightarrow \infty}-\frac{1}{N} \log \mathbb{P}_{H_{1}}\left(T_{N}^{(i)}<\gamma_{N}\right)=b .
\end{aligned}
$$

We denote by $\mathcal{S}_{i}$ the set of achievable pairs of error exponents for test $T_{N}^{(i)}$ and we refer to this set as the error exponent curve.

\section{IV-B. Main Result}

In order to express the error exponents of interest, we need further notations. Recall that the limiting probability distribution of the empirical distribution of the eigenvalues $F_{N}(x)=\frac{\#\left\{i, \lambda_{i} \leq x\right\}}{K}$ of $\hat{\mathbf{R}}=\frac{1}{N} \mathbf{Y} \mathbf{Y}^{H}$ is (under both assumptions $H_{0}$ or $H_{1}$ ) MarčhenkoPastur distribution:

$$
\mathbb{P}_{\check{M} P}(d y)=\mathbf{1}_{\left(\lambda^{-}, \lambda^{+}\right)}(y) \frac{\sqrt{\left(\lambda^{+}-y\right)\left(y-\lambda^{-}\right)}}{2 \pi c y} d y,
$$

where $\lambda^{+}=(1+\sqrt{c})^{2}$ and $\lambda^{-}=(1-\sqrt{c})^{2}$. We also introduce $\lambda_{\mathrm{spk}}^{\infty}=(1+\rho)\left(1+\frac{c}{\rho}\right)$ (recall that the largest eigenvalue $\lambda_{1}$ converges toward $\lambda^{+}$under $H_{0}$ and toward $\lambda_{\mathrm{spk}}^{\infty}$ under $H_{1}$ - see for instance [8]). Of prime importance is the Stieltjes transform of $\mathbb{P}_{\check{M} \text { MP }}, \mathbf{f}(x)=\int \frac{\mathbb{P}_{\check{M} \mathrm{P}}(d y)}{y-x}$ which admits the following well-known closed-form representations:

$$
\mathbf{f}(x)=\frac{(1-x-c)+\epsilon_{x} \sqrt{(1-x-c)^{2}-4 c x}}{2 c x}
$$

where $\epsilon_{x}=1$ if $x>\lambda^{+}$and $\epsilon_{x}=-1$ if $x \in\left(0, \lambda^{-}\right)$. Define:

$$
\begin{cases}\mathbf{F}^{+}(x)=\int \log (x-y) \mathbb{P}_{\check{\mathrm{MP}}}(d y) & \text { for } x>\lambda^{+}, \\ \mathbf{F}^{-}(x)=\int \log (y-x) \mathbb{P}_{\check{\mathrm{MP}}}(d y) & \text { for } x \in\left(0, \lambda^{-}\right)\end{cases}
$$

and let $\tilde{\mathbf{f}}(x)=-\frac{1}{x(1+c \mathbf{f}(x))}$.

Lemma 1. The following representations hold true:

$$
\begin{aligned}
\mathbf{F}^{+}(x)= & \log (x)+\frac{1}{c} \log (1+c \mathbf{f}(x))+\log (1+\tilde{\mathbf{f}}(x)) \\
& +x \mathbf{f}(x) \tilde{\mathbf{f}}(x) \\
\mathbf{F}^{-}(x)= & \log (x)+\frac{1}{c} \log (1+c \mathbf{f}(x))+\log (-(1+\tilde{\mathbf{f}}(x))) \\
& +x \mathbf{f}(x) \tilde{\mathbf{f}}(x) .
\end{aligned}
$$

For similar computations, see for example [9, Section 4]. We are now in position to introduce the functions that will help to express the error exponents. Denote by $\Delta(\cdot \mid A)$ the convex indicator function defined by:

$$
\Delta(x \mid A)= \begin{cases}0 & \text { if } x \in A \\ \infty & \text { else }\end{cases}
$$


Define for each $\rho>\sqrt{c}$ :

$$
\begin{aligned}
I_{\rho}^{+}(x)= & \frac{x-\lambda_{\mathrm{spk}}^{\infty}}{(1+\rho)}-(1-c) \log \left(\frac{x}{\lambda_{\mathrm{spk}}^{\infty}}\right) \\
& \quad-c\left(\mathbf{F}^{+}(x)-\mathbf{F}^{+}\left(\lambda_{\mathrm{spk}}^{\infty}\right)\right)+\Delta\left(x \mid\left[\lambda^{+}, \infty\right)\right), \\
I_{0}^{+}(x)= & x-\lambda^{+}-(1-c) \log \left(\frac{x}{\lambda^{+}}\right) \\
& \quad-2 c\left(\mathbf{F}^{+}(x)-\mathbf{F}^{+}\left(\lambda_{\mathrm{spk}}^{\infty}\right)\right)+\Delta\left(x \mid\left[\lambda^{+}, \infty\right)\right), \\
I^{-}(y)=\quad y-\lambda_{\mathrm{spk}}^{\infty}-(1-c) \log \left(\frac{y}{\lambda_{\mathrm{spk}}^{\infty}}\right) & -2 c\left(\mathbf{F}^{-}(y)-\mathbf{F}^{-}\left(\lambda^{-}\right)\right)+\Delta\left(y \mid\left(0, \lambda^{-}\right]\right) .
\end{aligned}
$$

As one may expect, $I_{\rho}^{+}$(resp. $I_{0}$ ) and $I^{-}$are associated to the Large Deviation Principle (LDP) governing $\lambda_{1}$ and $\lambda_{K}$ respectively when $\rho>\sqrt{c}$ (resp. $\rho=0$ ). Define $\Gamma_{\rho}(t)=$ $\inf \left\{I_{\rho}^{+}(x)+I^{-}(y), \quad \frac{x}{y}=t\right\}$ for $\rho>\sqrt{c}$ and define $\Gamma_{0}$ similarly. It can be shown that $\Gamma_{\rho}$ and $\Gamma_{0}$ are associated to the LDP governing $\lambda_{1} / \lambda_{K}$ when $\rho>\sqrt{c}$ and $\rho=0$ respectively.

Theorem 1. Assume that $\rho>\sqrt{c}$. Error exponents $\mathcal{E}_{1, \rho}$ and $\mathcal{E}_{2, \rho}$ are well defined and are given by:

$$
\mathcal{E}_{1, \rho}=\mathcal{E}_{2, \rho}=I_{\rho}^{+}\left(\lambda^{+}\right) .
$$

The error exponent curves of both tests are given by:

$$
\begin{aligned}
& \mathcal{S}_{1}=\left\{\left(I_{0}^{+}(x), I_{\rho}^{+}(x)\right): x \in\left(\lambda^{+}, \lambda_{\mathrm{spk}}^{\infty}\right)\right\} \\
& \mathcal{S}_{2}=\left\{\left(\Gamma_{0}(x), \Gamma_{\rho}(x)\right): x \in\left(\lambda^{+} / \lambda^{-}, \frac{\lambda_{\mathrm{spk}}^{\infty}}{\lambda^{-}}\right)\right\} .
\end{aligned}
$$

In particular, the error exponent curve $\mathcal{S}_{1}$ uniformly dominates $\mathcal{S}_{2}$ in the sense that for each $(a, b) \in \mathcal{S}_{2}$ there exits $b^{\prime}>b$ such that $\left(a, b^{\prime}\right) \in \mathcal{S}_{1}$.

The proof will be provided in an extended version of this paper.

\section{IV-C. Comments and Numerical Results}

In terms of error exponents, both tests $T_{N}^{(1)}$ and $T_{N}^{(2)}$ admit the same error exponent as long as the level $\alpha$ of the test is kept fixed. Furthermore, error exponents do not depend on $\alpha$. Figure 2 represents the error exponent of both tests in log-scale as a function of the SNR $\rho$ in $\mathrm{dB}$. Error exponents are compared with the error exponent associated with the Neyman-Pearson test, achieved in the ideal case where parameters $\mathbf{H}$ and $\sigma$ are known. The error exponent of the Neyman-Pearson test can be derived from Stein's Lemma, and provides an upper bound on the achievable error exponents. Note that when $\rho<\sqrt{c}$, the test statistic $T_{N}^{(1)}$ converges to the same limit under $H_{0}$ and under $H_{1}$. A similar behaviour occurs for $T_{N}^{(2)}$ due to equation (13). Thus, both tests fail when $\rho<\sqrt{c}$. Therefore, it is not surprising that the error exponent tends to zero when $\rho$ is close to $\sqrt{c}$.

Figure 3 represents the error exponent curves $S_{1}$ and $S_{2}$ for $\rho=$ 1 and $c=0.5$. As stated by Theorem 1, the error exponent curve associated with test $T_{N}^{(1)}$ uniformly dominates the one associated with $T_{N}^{(2)}$.

\section{REFERENCES}

[1] M. Wax and T. Kailath. Detection of signals by information theoretic criteria. IEEE Trans. on Signal, Speech, and Signal Processing, 33(2):387-392, April 1985

[2] J. Mitola. Cognitive Radio An Integrated Agent Architecture for Software Defined Radio. PhD thesis, Royal Institute of Technology (KTH), May 2000.

[3] L. S. Cardoso, M. Debbah, P. Bianchi, and J. Najim. Cooperative Spectrum Sensing Using Random Matrix Theory. 2008.

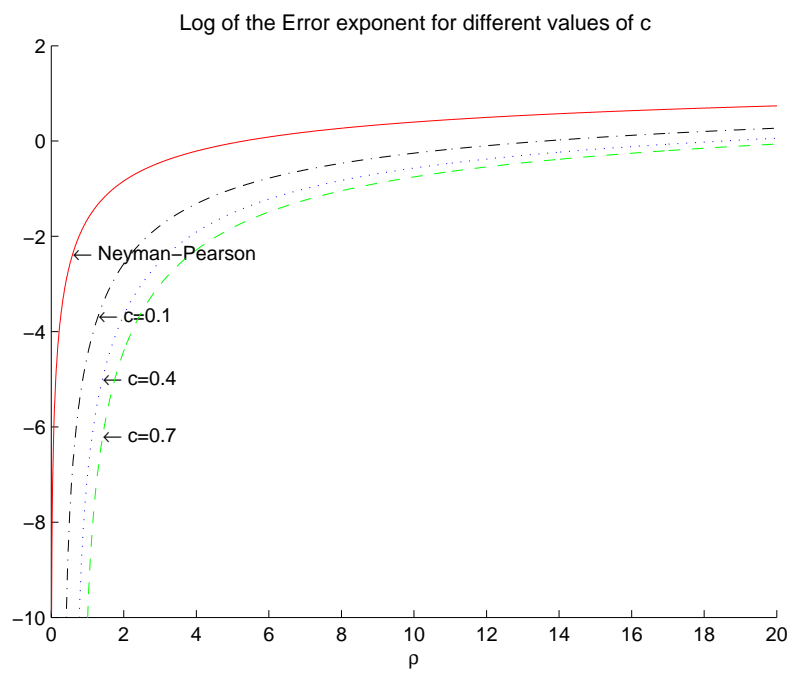

Fig. 2. Logarithm of the error exponent $\mathcal{E}_{\rho}$ as a function of $\rho$ (in $\mathrm{dB})$ for different values of $c$.

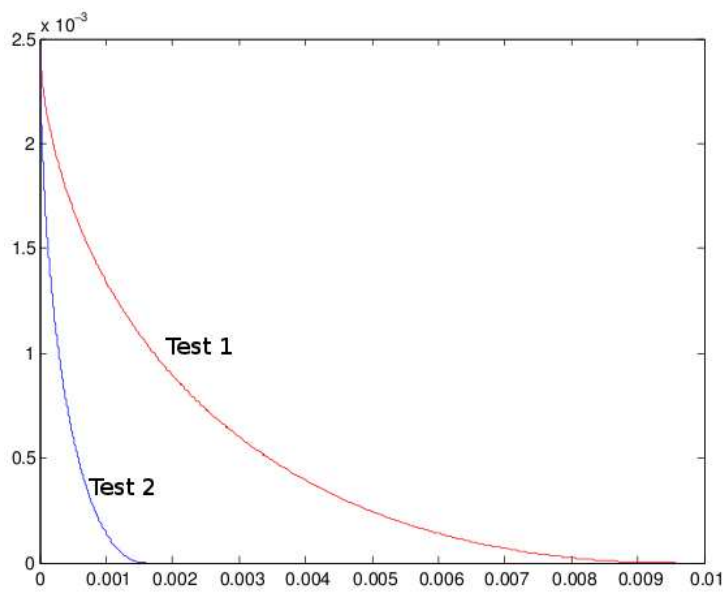

Fig. 3. Error exponent curves $\mathcal{S}_{1}$ and $\mathcal{S}_{2}$ associated with both tests $T_{N}^{(1)}$ and $T_{N}^{(2)}-\rho=1-c=0.5$.

[4] Y. H. Zeng and Y. C. Liang. Eigenvalue based spectrum sensing algorithms for cognitive radio. to appear in IEEE Trans on Communications volume $=$ arXiv:0804.2960v1.

[5] H. Urkowitz. Energy detection of unknown deterministic signals. Proceedings of the IEEE, 55:523-531, 1967.

[6] P. Bianchi, J. Najim, G. Alfano, and M. Debbah. Asymptotics of eigenbased collaborative sensing. In submitted to Information Theory Workshop ITW, 2009.

[7] F. Penna, R. Garello, and M. A. Spirito. Cooperative Spectrum Sensing based on the Limiting Eigenvalue Ratio Distribution in Wishart Matrices. IEEE Communication Letters, submitted, 2009.

[8] J. Baik and J. Silverstein. Eigenvalues of large sample covariance matrices of spiked population models. J. Multivariate Anal., 97(6):13821408, 2006.

[9] W. Hachem, P. Loubaton, and J. Najim. Deterministic equivalents for certain functionals of large random matrices. Ann. Appl. Probab., 17(3):875-930, 2007. 